\title{
Analysis of Flame Deflector Spray Nozzles in Rocket Engine Test Stands
}

\author{
Jai S. Sachdev ${ }^{1}$, Vineet Ahuja ${ }^{2}$, Ashvin Hosangadi ${ }^{3}$ \\ Combustion Research and Flow Technology Inc., Pipersville, PA 18947 \\ and \\ Daniel C. Allgood ${ }^{4}$ \\ NASA Stennis Space Center, Stennis Space Center, MS 39529
}

\begin{abstract}
The development of a unified tightly coupled multi-phase computational framework is described for the analysis and design of cooling spray nozzle configurations on the flame deflector in rocket engine test stands. An Eulerian formulation is used to model the disperse phase and is coupled to the gas-phase equations through momentum and heat transfer as well as phase change. The phase change formulation is modeled according to a modified form of the Hertz-Knudsen equation. Various simple test cases are presented to verify the validity of the numerical framework. The ability of the methodology to accurately predict the temperature load on the flame deflector is demonstrated though application to an actual sub-scale test facility. The CFD simulation was able to reproduce the result of the test-firing, showing that the spray nozzle configuration provided insufficient amount of cooling.
\end{abstract}

\section{Introduction}

$\mathrm{N}$ ASA Stennis Space Center (SSC) serves as NASA's lead facility for conducting large-scale liquid rocket engine tests. Typically, engine or rocket stage testing consists of mounting the engine/stage vertically on a test stand where the exhaust plume fires down into a plume deflector. The deflector then serves to redirect the exhaust flow safely away from the test facility as shown in Figure 1. One of the key challenges of conducting long duration static rocket tests is protecting the deflector from harsh thermal environments generated by plume impingement. For example, during the Space Shuttle Main Engine (SSME) test program the nominal burn time was 480 seconds, and the longest static SSME test ever conducted was for $2017 \mathrm{sec}$ in 1988 on the B-1 test stand. ${ }^{1}$ Thus, there is sufficient time for the deflector steel structure to reach a thermal equilibrium.

Currently, in a majority of cases, the flame deflectors on test stands at Stennis are cooled by water sprays, where large flow rates of water (up to $300,000 \mathrm{gpm}$ ) is pumped by a highpressure industrial water facility to the test complexes and then injected through thousands of small diameter holes along the deflector surface. Historically, the process of designing the placement of these water cooling holes have been based on simplified analytical estimates of plume impingement dynamics on the deflector and its associated heat transfer. In these analytical calculations, an un-cooled or "dry" rocket plume was assumed. More recently, the simple 1-D or quasi 2-D analytical tools have been replaced with single-phase CFD analysis to achieve more accurate estimations of un-

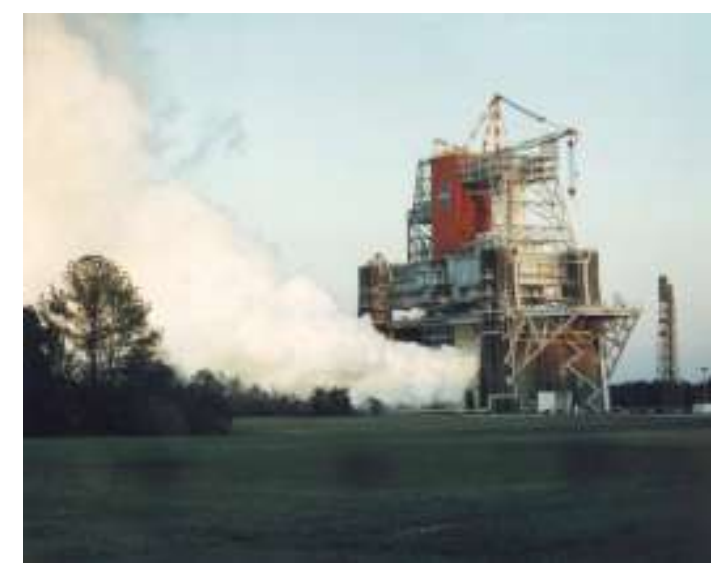

Figure 1: Space Shuttle Main Engine Test at the NASA Stennis B-1 Test Stand.

\footnotetext{
${ }^{1}$ Research Scientist, AIAA Member.

${ }^{2}$ Senior Research Scientist, Senior AIAA Member.

${ }^{3}$ Principal Scientist, Senior AIAA Member.

${ }^{4}$ Senior Scientist, Senior AIAA Member.
} 
cooled heating rate distributions. ${ }^{2}$ Ultimately, the water cooling requirements are derived by correlating the local un-cooled plume heating rate to water cooling flow rates using "experience curves". The experience curves consist of previous successful tests where a linear relationship between the water flow rate density and un-cooled heating rate has been assumed. A dimensional analysis can be performed along with an energy balance to show that the slope of the experience curve is inversely proportional to the product of the heat of evaporation and density of the water. The resulting proportionality constant of this linear relation is defined as the efficiency of the water cooling, where $100 \%$ efficiency assumes all the water evaporates and goes into cooling the deflector wall. In reality, not all the water successfully participates in removing the heat from the wall through evaporative cooling. In reality, the cooling efficiency is dependent upon geometric parameters such as engine-deflector separation, plume impingement angle, cooling hole spacing, hole size, injection angles, etc. In addition to these key geometrical parameters, the effect of engine operating conditions and propellant types can also be important. Clearly, there is a large uncertainty in this engineering design approach due to the lack of knowledge on deflector water cooling and the lack of validated modeling tools to capture non-equilibrium multi-phase flow of water sprays in a supersonic rocket plume environment.

In this paper, we present a unified tightly coupled multi-phase computational framework that will be used to analyze both the "wet plume" emanating from the rocket engines as well as predict the surface temperature on the flame deflector that results from the interaction of the plume and water from the cooling holes/nozzles. Since the plume flowfield and the water droplets from the cooling nozzles are in non-equilibrium a dispersed phase model has been added to our existing CFD framework. The formulation of this model is discussed in Section II along with the mechanism for phase change. The implementation of the model and the numerical framework are discussed in Section III. Fundamental validation studies for droplet phase change and the cooling of a heated plate by a liquid spray are shown in Sections IV and V. Finally engine exhaust simulations impinging on a flame deflector that is cooled with a water spray are discussed in Section VI.

\section{Droplet Modeling for Flame Deflector Spray Nozzles}

Cooling of the flame deflector is achieved by the injection of water through an array of nozzles. To accurately model the non-equilibrium effects of the impinging plume and the injected water spray, the computational framework has been extended to include an Eulerian formulation for a dispersed droplet phase. This requires the solution of an additional set of conservation for the droplet phase and the two phases are coupled through momentum and heat transfer as well as mass transfer through evaporation/condensation. The droplet formulation is based on the work of Kenzakowski and Brinckman. ${ }^{3}$

\section{A. Droplet Phase Conservation Equations}

The equations governing the behavior of the droplet cloud are

$$
\frac{\partial Q_{p}}{\partial t}+\frac{\partial E_{p}}{\partial x}+\frac{\partial F_{p}}{\partial y}+\frac{\partial G_{p}}{\partial z}=D_{p}+H_{p}
$$

where the vector $Q_{p}$ is the vector of dependent variables and the vectors $E_{p}, F_{p}, G_{p}$ are the convective fluxes. These vectors are given by

$$
Q_{p}=\left[\begin{array}{c}
\rho_{p} \\
\rho_{p} u_{p} \\
\rho_{p} v_{p} \\
\rho_{p} w_{p} \\
h_{p} \\
S_{p}
\end{array}\right] ; \quad E_{p}=\left[\begin{array}{c}
\rho_{p} U_{p} \\
\rho_{p} U_{p} u_{p} \\
\rho_{p} U_{p} v_{p} \\
\rho_{p} U_{p} w_{p} \\
h_{p} U_{p} \\
S_{p} V_{p}
\end{array}\right] ; \quad F_{p}=\left[\begin{array}{c}
\rho_{p} V_{p} \\
\rho_{p} V_{p} u_{p} \\
\rho_{p} V_{p} v_{p} \\
\rho_{p} V_{p} w_{p} \\
h_{p} V_{p} \\
S_{p} V_{p}
\end{array}\right] ; \quad G_{p}=\left[\begin{array}{c}
\rho_{p} W_{p} \\
\rho_{p} W_{p} u_{p} \\
\rho_{p} W_{p} v_{p} \\
\rho_{p} W_{p} w_{p} \\
h_{p} W_{p} \\
S_{p} W_{p}
\end{array}\right]
$$

For the droplet phase, $\rho_{p}=4 \pi \rho_{\text {liq }} N_{p} r_{\text {smd }}{ }^{3} / 3$ and $S_{p}=4 \pi N_{p} r_{\text {smd }}{ }^{2}$ represent the droplet cloud density and the net exposed surface area per unit volume of the droplet cloud, respectively. Where $N_{p}$ is the droplet number density and $r_{\text {smd }}$ is the Sauter mean radius. The Sauter mean radius can be determined from $r_{\text {smd }}=3 \rho_{p} /\left(S_{p} \rho_{\text {liq }}\right)$ where $\rho_{\text {liq }}$ 
is the physical thermodynamic density of the liquid. These equations are solved in addition to the full compressible Navier-Stokes equations. The two phases are coupled through the momentum and heat transfer source term vector, $D_{p}$, as well as the phase change source term, $H_{p}$. These vectors are given by

$$
D_{p}=\frac{1}{V_{\text {cell }}}\left[\begin{array}{c}
0 \\
A_{p}\left(u_{g}-u_{p}\right) \\
A_{p}\left(v_{g}-v_{p}\right) \\
A_{p}\left(w_{g}-w_{p}\right) \\
B_{p}\left(T_{g}-T_{p}\right) \\
0
\end{array}\right] ; \quad H_{p}=\frac{1}{V_{\text {cell }}}\left[\begin{array}{c}
\dot{m}_{p} \\
\dot{m}_{p} u_{p} \\
\dot{m}_{p} v_{p} \\
\dot{m}_{p} w_{p} \\
\dot{m}_{p} h_{\text {vap }} \\
2 \dot{m}_{p} /\left(r_{\text {smd }} \rho_{\text {liq }}\right)
\end{array}\right]
$$

The momentum and heat transfer terms, $A_{p}$ and $B_{p}$, functions of the drag coefficient, $\left(\mathrm{C}_{\mathrm{d}}\right)$, and the Nusselt number $(\mathrm{Nu})$ and are defined as.

$$
A_{p}=\frac{3}{8} \frac{\rho}{\rho_{p} r_{\text {smd }}} C_{d}\left|\vec{U}-\vec{U}_{p}\right| ; \quad B_{p}=\frac{3}{8} \frac{\mu}{\rho_{p} r_{\text {smd }}^{2}} \frac{C_{p}}{P_{r}} N u
$$

The current formulation utilizes correlations derived from Henderson and Hermsen for rocket propulsion applications. $^{4,5}$

\section{B. Droplet Enthalpy-Temperature Relation}

Closure of the droplet cloud equations was obtained by using a polynomial fit to the NIST data set to determine the droplet enthalpy, $h_{p}$, as a function of its temperature. An additional curve fit was used to compute the droplet temperature given its enthalpy. An alternative approach was also implemented to determine the enthalpy from the droplet's temperature and pressure based on the HBMS equation of state ${ }^{6}$. However, this method required an iterative solution to find the droplet's temperature given its enthalpy rendering it more computationally expensive. Therefore, the first method was used in this work, although the second method will be useful when modeling fuel droplets at cryogenic temperatures.

\section{Droplet Phase-Change Modeling}

The rate of mass transfer due to evaporation and condensation, $\dot{m}_{p}$, is modeled according to a modified form of the Hertz-Knudsen equation. This relation gives the mass transfer rate as the difference between incoming (condensing) fluxes from the gas phase, and outgoing (evaporative) fluxes from the droplet.

$$
\dot{m}_{p}=4 \pi N_{p} r^{2}\left[\frac{P_{\text {partial }}}{\sqrt{2 \pi R T}}-\frac{P_{\text {sateff }}}{\sqrt{2 \pi R T_{p}}}\right]
$$

The partial pressure of the droplet substance vapor in the gas phase is given by $P_{\text {partial }}$. The modification to the Hertz-Knudsen equation is that an effective saturation pressure, $P_{\text {sateff }}$, given by the Kelvin-Helmholtz equation is used. This formulation accounts for surface tension, $\sigma$, and is given by

$$
P_{\text {sateff }}=P_{\text {sat }} \exp \left[\frac{2 \sigma}{\rho_{\text {liq }} R T_{p} r_{\text {smd }}}\right]
$$

Here $P_{s a t}$ is the flat film saturation pressure at the droplet temperature. Surface tension is also expressed as a function of drop temperature. The source term for the energy equation is obtained from the mass transfer rate, $\dot{m}_{p}$, and the difference of enthalpy between the two phases, $h_{\text {vap }}$ (heat of vaporization). Therefore, during condensation the enthalpy of the gas phase is transferred to the liquid and the droplets tend to warm up. Upon vaporization the

American Institute of Aeronautics and Astronautics 
enthalpy of the gas phase evaluated at the droplet temperature is transferred from the liquid to the gas and the droplet cools with net vaporization.

\section{Numerical Framework for Flame Deflector Spray Nozzles}

\section{A. $C R U N C H C F D^{\circledR}$ Numerical Framework}

The CRUNCH CFD ${ }^{\circledR}$ code is a multi-element (i.e. tetrahedral, prismatic, pyramid, and hexahedral cells) unstructured flow solver for viscous, real gas systems ${ }^{7-9}$. It is formulated for an edge-based data framework where the solution is saved at the cell-vertex and a dual control-volume is defined by cutting across all edges coming to a node. ${ }^{10,11}$ Such an edge-based formulation is attractive when dealing with multi-elements since the dual surface associated with an edge can include contributions from different element types resulting in a "grid transparent" framework for inviscid flows.

The governing equations for the conservation of mass, momentum, energy, species continuity, and k-epsilon turbulence model variables are solved with finite-volume scheme for each vertex dual control-volume. The 'standard' high Reynolds number form of the k-epsilon equations forms the basis of the turbulence modeling used in this work. Solution of the species continuity equations allow for the analysis of generalized, multi-component gaseous flow fields including real gas behavior. Non-equilibrium combustion phenomenon can be modeled using finite rate chemical kinetic source terms in a completely general fashion where an arbitrary set of reactions may be specified by the user. The reader is referred to Ref. 8 for more details on the combustion modeling approach implemented in $C R U N C H C F D^{\circledR}$.

The inviscid flux procedure involves looping over the edge list and computing the flux at the dual face area bisecting the edge. A Riemann problem is solved for using higher order reconstructed values at the dual face. ${ }^{9}$ Presently, a second-order linear reconstruction procedure is employed to obtain a higher order scheme. ${ }^{10,11}$ The higher-order variables need to be limited to yield a total variation diminishing (TVD) method. We note that the inviscid flux procedure as outlined here is grid transparent in that the details of the different element types contributing to a dual face area edge are not relevant.

The viscous fluxes are computed by estimating the gradients (or stresses) at the cell faces and then performing a Green-Gauss integration of the stresses around each dual control volume. ${ }^{9}$ For hexahedral cells where the edge vectors are not skewed relative to the cell faces, an edge based viscous flux procedure can be derived. However, for tetrahedral cells where edge skewness is substantial, the edge based procedure fails, particularly for the k-epsilon equations, and is reflected in incorrect turbulent viscosity levels. For tetrahedral cells, a cell based procedure has been implemented which remedies this problem.

For efficient computation of large 3D problems, a parallel framework for distributed memory systems has been implemented along with an implicit solution procedure for the sparse implicit Jacobian matrix. The parallel framework is implemented by partitioning the grid into sub-domains with each sub-domain residing on an independent processor. Since the solution is solved for at the cell vertex, the control volume for the nodes lying on the inter-processor boundaries may span across processors. The net flux for the inter-processor nodes are obtained by summing up the fluxes computed independently on each processor and hence the explicit flux is identical to the single-processor solution. The message passing between processors has been implemented using MPI to provide portability across various platforms.

The implicit solution procedure allows for Gauss-Seidel or generalized minimal residual (GMRES) solver options with a preconditioning matrix using distance-one neighbor bandwidth. ${ }^{11}$ Currently, the approximate Jacobian for the Roe-average flux is taken and this together with the sparseness of the preconditioning matrix may affect the stability of the inversion procedure. The implicit inversion process is implemented for parallel, partitioned grids by summing up the diagonal blocks of the sparse matrix for inter-processor nodes. The off-diagonal blocks for inter-processor nodes can be made zero to ensure identical solutions on each processor. In this case, the stability of the procedure would depend on the diagonal dominance of the system. Conversely, if the off-diagonal blocks were not made zero, the solution on inter-processor nodes would need to be averaged across processors to obtain a common solution on all processors.

\section{B. Extension of Numerical Framework for the Droplet Phase Equations}

The equations described in Section II govern the motion of a dilute and disperse droplet-phase indicating that the volume fraction of the droplet cloud is negligible and that inter-particle collisions are not important. The resulting set of equations is very similar to the Eulerian equations for inviscid, compressible gases with the exception of the absence of a pressure-like terms in the droplet-phase momentum and energy equations. The similarity of these equations to the Euler equations provides a distinct advantage of using an Eulerian formulation for the droplet-phase 
versus a Lagrangian formulation in that the resulting set of governing equations can be solved using the same numerical framework described above for the fluid equations. Enhanced diffusion of the droplet cloud due to turbulence is not considered in this work, therefore, only an upwind inviscid-flux procedure is required to model the convective fluxes. ${ }^{12-16}$

Although seemingly straight-forward, the mathematical nature of the droplet phase equations lead to limitations in the accuracy of the numerical simulation. In particular, it is well known that this system of equations is both hyperbolic and degenerate. ${ }^{13,14,17}$ The degeneracy is a direct result of the assumptions that the droplet cloud is dilute and disperse. A lack of particle collisions means that there can be no pressure-like term in the momentum and energy equations. Therefore, regions of particle vacuums can exist in the flow since there is no pressure force to drive particles from regions of high concentrations to low concentrations. A second implication of the degeneracy is that the paths of particles can cross. For example, fast moving particles can over-take slower moving particles without any interaction occurring between the two groups. A more complete description of the degeneracy and its implications are discussed in Ref. 17.

In this work, a simple upwind inviscid flux procedure is used to model the droplet-phase flux term. This method is able to account for the degeneracy by allowing for particle-phase vacuums to exist in the flow-field, however, flows involving particle compression waves, such as at reflection boundary conditions, are problematic and can lead to an over prediction of the droplet cloud concentration in those regions. This effect has also been reported by other researchers. ${ }^{13-16}$ A method for alleviating this effect was described in Ref. 15. Particle compression waves are accurately modeled with this scheme and, therefore, crossing trajectories and reflection boundary conditions can be realistically modeled. This method comes with a penalty in terms of memory and computation time and is, therefore, not pursued here.

\section{Validation Study: One-dimensional Convection of Water Droplets}

The convection of water droplets through a one-dimensional tube was considered as a first validation of the evaporation and condensation formulation described above. Here, a coupled gas-droplet flow was injected into a tube at a speed of $200 \mathrm{~m} / \mathrm{s}$. The two phases were injected out of thermodynamic equilibrium but will equilibrate as they travel through the domain. It should be noted that the path to equilibrium is influenced by the evaporation/condensation process as well as heat transfer.

\section{A. Net Evaporation}

In the first simulation the gas and droplets were injected at $300 \mathrm{~K}$ and $350 \mathrm{~K}$, respectively. The gas phase consisted of dry air at atmospheric pressure. The droplets were injected with a radius of one micrometer. Profiles of the gas and droplet temperatures, droplet radius, and Hertz-Knudsen terms are shown in Figure 2. Under these conditions, the droplets evaporate as they travel down the tube. From the profiles of the Hertz-Knudsen terms it can be seen the evaporation term dominates the mass transfer balance at the beginning. This is reflected in the temperature profile as there is a net loss in temperature. However, heat transfer quickly begins to dominate the thermal equilibrium process and the gas and droplet temperatures are both slightly below $300 \mathrm{~K}$ at the exit.

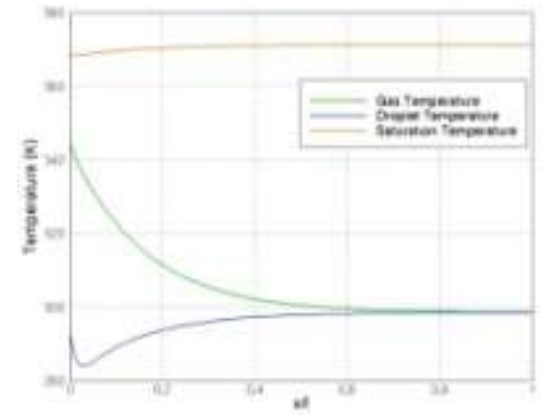

(a)

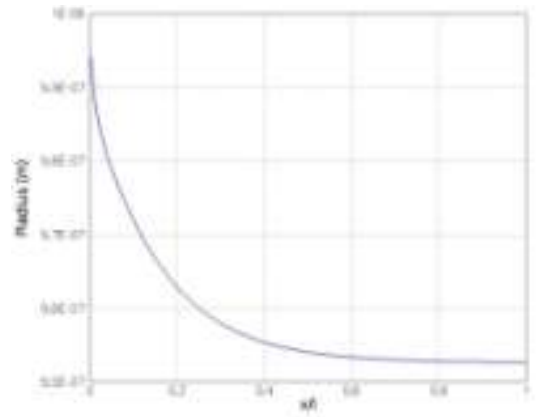

(b)

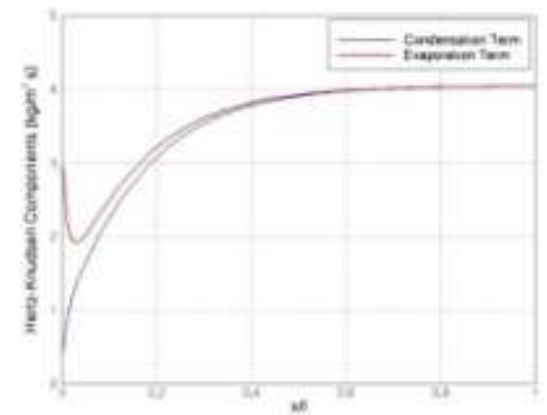

(c)

Figure 2: Profiles of the gas and droplet temperatures, droplet radius, and Hertz-Knudsen terms for a one-dimensional flow with net evaporation. 


\section{B. B. Net Condensation}

The second simulation was configured to result in a net condensation. Here the gas included $6.74 \%$ mass fraction of water vapor. The gas and droplet phases were injected at $320 \mathrm{~K}$ and $300 \mathrm{~K}$, respectively. From the profiles included in Figure 3, it can be seen that upon injection the droplets condensed quite rapidly followed by evaporation until thermodynamic equilibrium was met. During the condensation phase the temperature of the droplets increased as the enthalpy of the gas phase was transferred to the droplets.

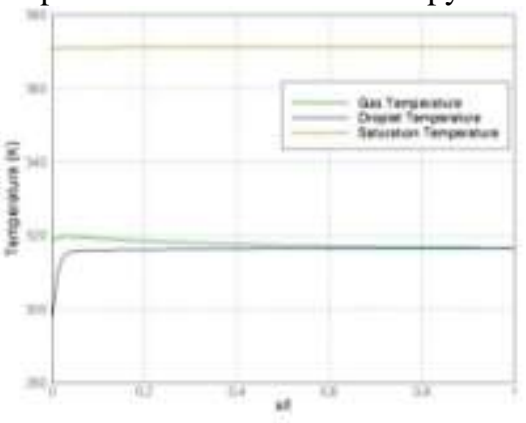

(a)

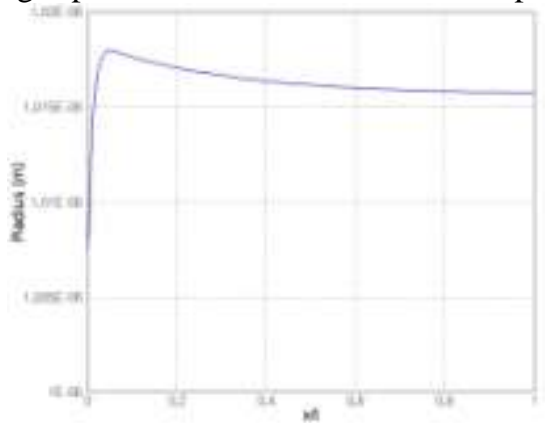

(b)

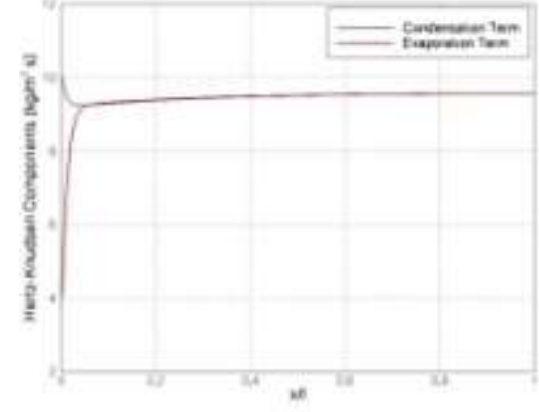

(c)

Figure 3: Profiles of the gas and droplet temperatures, droplet radius, and Hertz-Knudsen terms for a one-dimensional flow with net condensation.

\section{Validation Study: Cooling of Heated Chamber By Liquid Water Sprays}

Ciofalo et al. performed an experimental investigation of the cooling effect of a water spray on a chamber heated by a hot wall. ${ }^{1}$ In this work, the mid-plane temperature of a heated material was measured as it was cooled by a liquid spray with varying nozzle characteristics in a closed room. This configuration provides an excellent case to demonstrate the capability of the gas-droplet formulation to deliver a cooling spray of water droplets. The initial configuration for the domain and the initial steadystate contours (before droplet injection) are shown in Figure 4. The plate is heated to $543 \mathrm{~K}$ and the other walls are held at $293 \mathrm{~K}$. Snap-shots of the temperature and water vapor contours at 1, 2, and $3 \mathrm{~ms}$ into the simulation are provided in Figure 4 and Figure 5, respectively. The water vapor is generated by the evaporation of the injected droplets. The mass flow rate and injection speed of the droplets was $0.03 \mathrm{~kg} / \mathrm{s}$ and $26 \mathrm{~m} / \mathrm{s}$. The initial droplet diameter was set to $0.005 \mathrm{~m}$. The effectiveness of the spray for cooling the domain is evident from the snap-shots in Figure 4 where the temperature in the vicinity of the plate is seen to drop substantially from the interaction of the cooling spray with the plate.

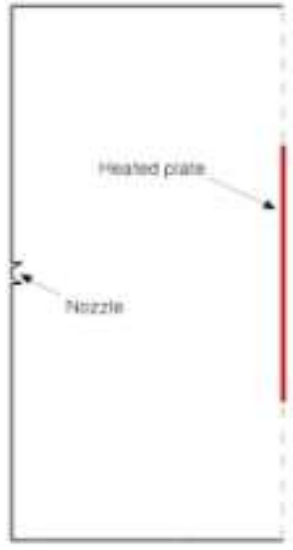

(a)

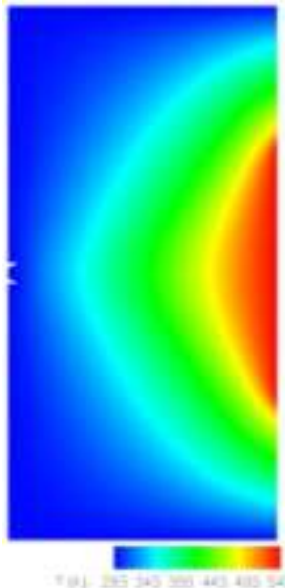

(b)

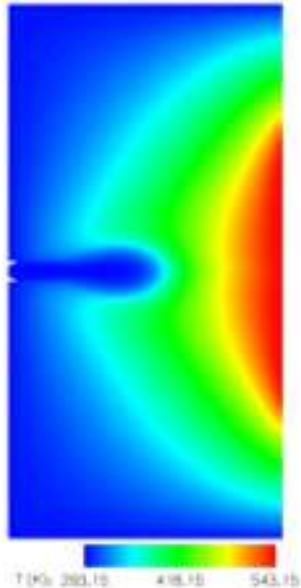

(c)

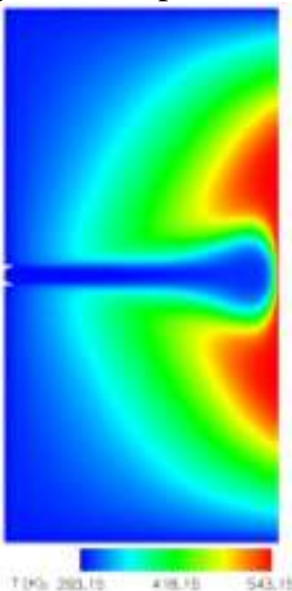

(d)

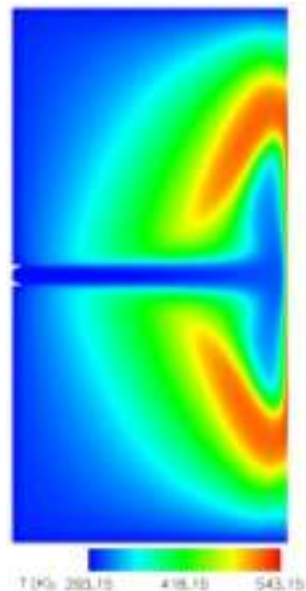

(e)

Figure 4: Chamber configuration (1st panel) and initial temperature contours (2nd panel). Panels 3-5 show snap-shots of the temperature contours at 1, 2, and $3 \mathrm{~ms}$ into the simulation. The cooling of the enclosed domain is evident. 


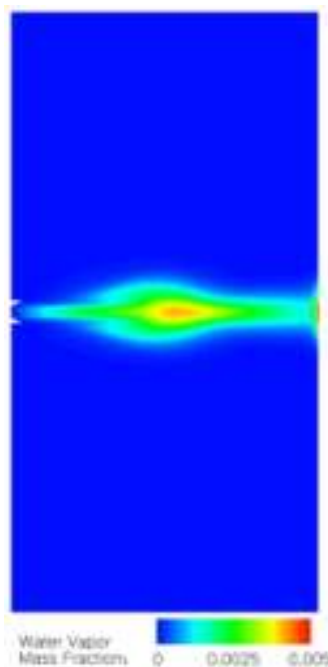

(a)

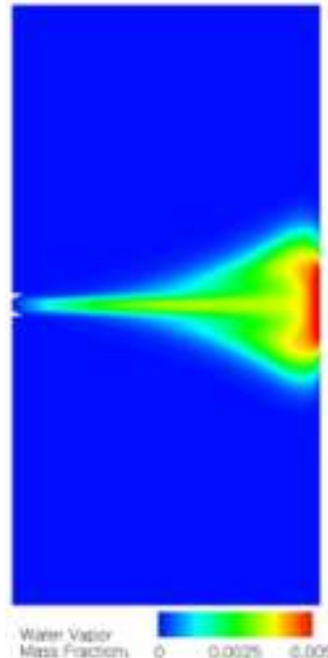

(b)

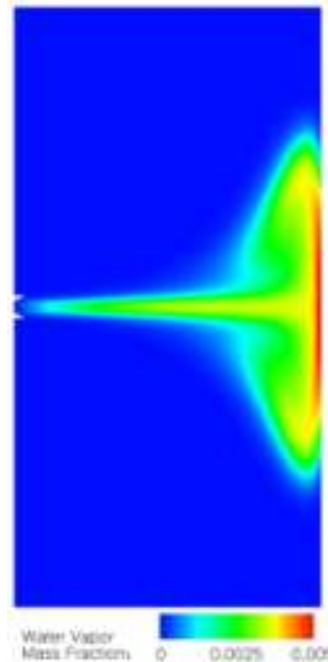

(c)

Figure 5: Snap-shots of the water vapor contours during 1, 2, and $3 \mathrm{~ms}$ into the simulation. The water vapor is generated by the evaporation of the injected water droplets.

\section{Flame Deflector Spray Nozzle Simulations}

A sub-scale test facility was designed and built at NASA SSC in order to experimentally assess the effectiveness of various spray nozzle configurations at keeping the flame deflector cool. A schematic of the facility can be seen in the left panel of Figure 6. Shown here are the rocket nozzle, the flame deflector plate, and the spray hole configuration that will be simulated in this work. It is noted that this is an extreme case such that the exit of the rocket was designed to sit very close to the flame deflector to deliver a maximum heat load. Holes 5/64 inches in diameter were drilled normally into the deflector panel and water delivery stagnation conditions in the manifold were 200 psi and 70 degrees F. Shown in the right panel of Figure 6 is a photograph of the result of the test firing for this configuration. It clearly shows where the water spray was able to keep the plate cool (rusted zones) and where it was unable to do so. The white sections of the plate show areas where the heat load was high enough to melt the flame deflector. Obviously, this configuration of spray nozzles provided insufficient cooling.

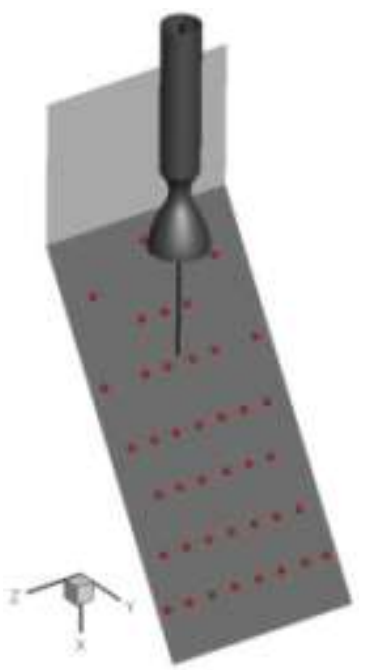

(a)

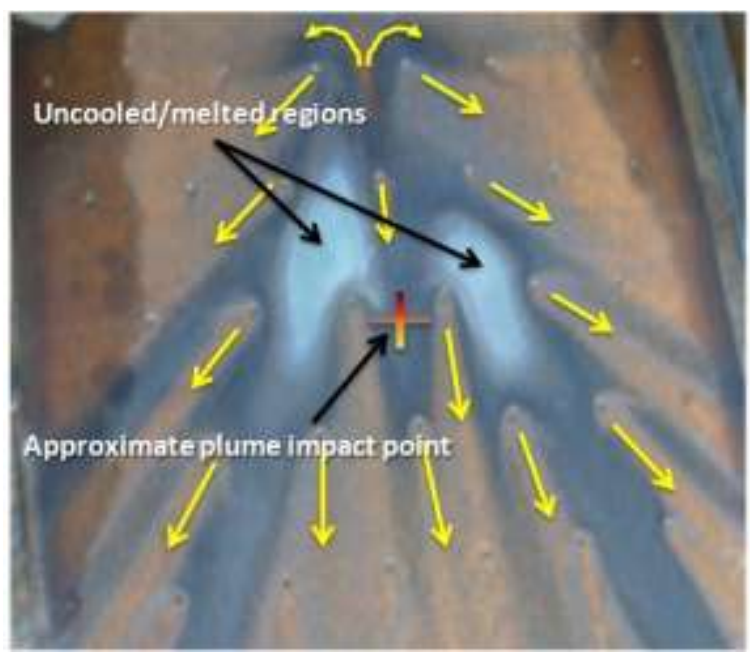

(b)

Figure 6: Left panel shows a schematic of the test facility including the spray hole configuration on the deflector plate. A photograph of the result of the experiment conduced at NASA SSC is shown on the right panel. Melted regions are clearly seen. 
In order to assess where the maximum temperature loads occur in the facility a "dry-plume" simulation was run in which no water cooling was used. The temperature, pressure, and Mach contours for this simulation are plotted on the deflector plate and symmetry plane in Figure 7. As expected, the region of maximum temperature and pressure load occurs directly under the rocket nozzle with temperatures and pressures in excess of $3000 \mathrm{~K}$ and 1 MPa. It can be seen from the Mach number contours that only a single shock cell is established before the plume impacts the deflector plate. A second shock cell-like structure is formed along the plate as the gas expands and compresses and terminates in a shock. A second high-temperature region occurs immediately after this shock wave. Temperature iso-surfaces of $1250 \mathrm{~K}$ and $2500 \mathrm{~K}$ are also provided in the fourth panel of Figure 7 . The two hot zones on the plate can be clearly seen as well as the plume dynamics as it impacts the deflector plate. It is also noted that the average temperature over the entire plate is over $1000 \mathrm{~K}$.

The results of the "wet plume" simulation in which the spray nozzles were activated can be seen in Figure 8 and Figure 9. It can be seen from the temperature and pressure contours plotted in Figure 8 that the current spray hole configuration does a reasonable job at relieving the heat and pressure loads on the deflector plate. The droplet plume effectively lifts the hot temperature zone directly below the rocket nozzle off of the plate. However, some hot gas is able to spill over the side of the droplet plume and raises the plate temperature to an extreme level. The hot plume travelling down the plate does not expand and compress as strongly as it did in the dry-plume simulation. Therefore, the droplet spray was able to completely eliminate the second hot zone. Iso-temperature and droplet cloud iso-density contours are given in Figure 9. The iso-temperature contours clearly show the lift off of the hotzone under the nozzle as well as the streaks of hot gas on the plate beside this area. The dynamics of the spray nozzle plumes can be seen from the droplet-cloud iso-density contours. In particular, one can see the channels of hot-gas and evaporated droplets that occur between large regions of high droplet concentration. The interaction between the spray nozzle plumes and rocket plume can be understood by the third panel of Figure 9 in which the two sets of iso-contours are plotted together. This figure clearly shows a region of hot gas from the rocket sitting above a plume of a spray nozzle. Moreover, first panels of Figure 8 and Figure 9 clearly show streaks of hot-gas on the deflector plate situated beside and slightly above the projected rocket plume impact zone. These temperature loads are sufficiently high enough to melt the deflector plate and predict exactly the locations of the melted regions found from the experimental test result as seen in the right panel of Figure 6. Therefore, the numerical framework developed for the analysis of flame deflector spray nozzle configurations was able to verify that the current spray hole configuration was unable to provide sufficient cooling.

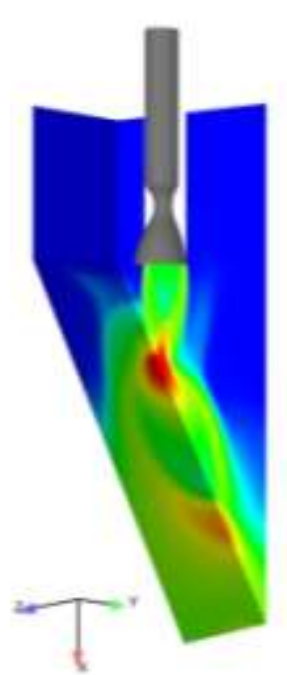

(a)

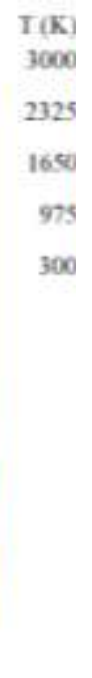

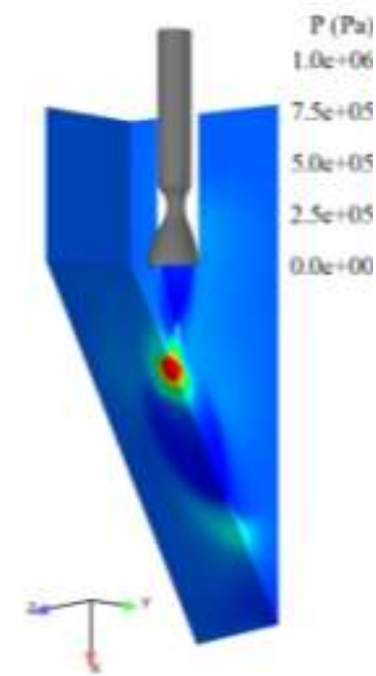

(b)

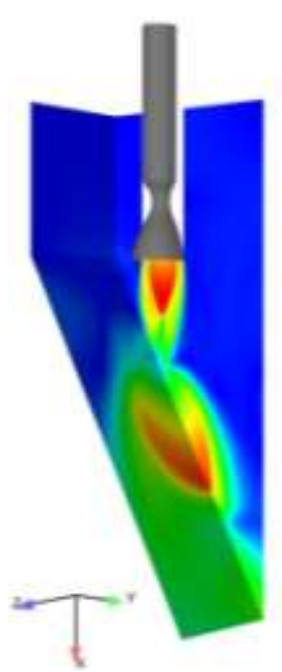

(c)

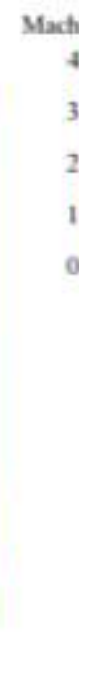

(2)

Figure 7: Temperature, pressure, and Mach number contours on the deflector plate and symmetry plane for the dry plume simulation (without cooling spray). Temperature iso-surfaces (1250 K and $2500 \mathrm{~K}$ ) are shown in the far right panel. Two regions of extreme heat loading can be seen. 


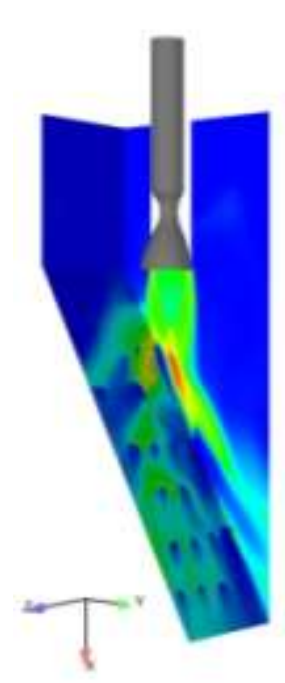

(a)

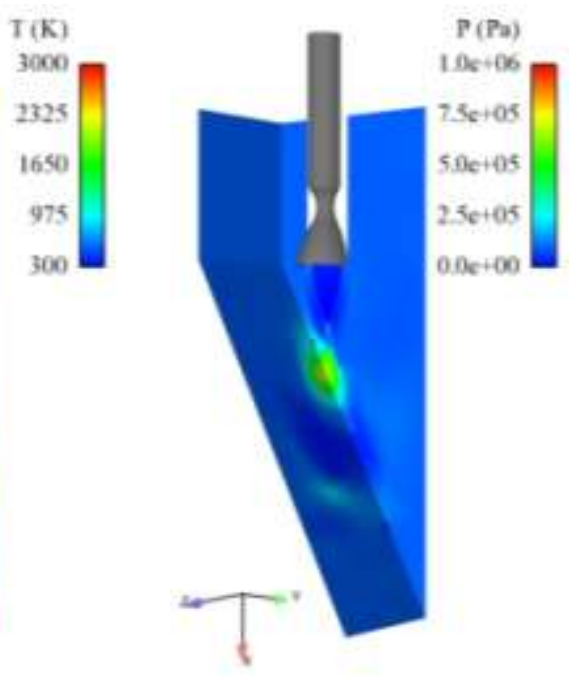

(b)

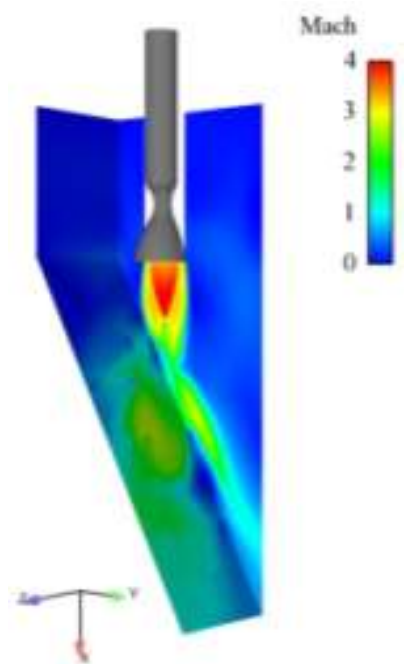

(c)

Figure 8: Temperature, pressure, and Mach number contours on the deflector plate and symmetry plane for the wet plume simulation (with cooling spray).
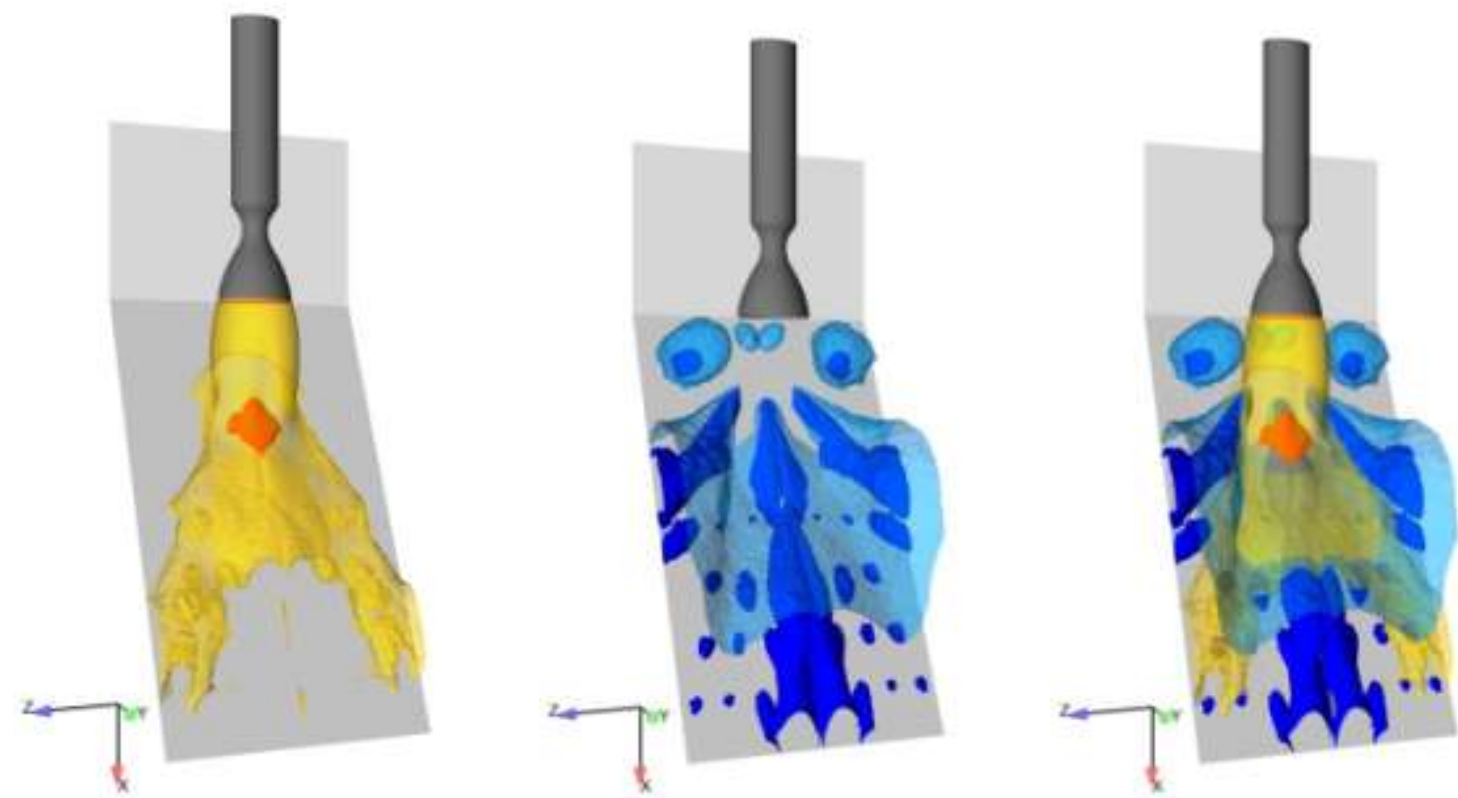

Figure 9: Wet-plume iso-temperature contours $(1250 \mathrm{~K}$ and $2500 \mathrm{~K})$ and droplet cloud iso-density contours $\left(0.5 \mathrm{~kg} / \mathrm{m}^{3}\right.$ and $\left.0.005 \mathrm{~kg} / \mathrm{m}^{3}\right)$ are shown in left and middle panels. An overlay of the two set of iso-contours is given on the right, showing the interaction of the rocket and spray nozzle plumes.

\section{Summary}

One of the most serious issues with rocket engine test facilities relates to heat loads on the facility flame deflector during rocket engine test firing. High temperatures associated with the plume and long duration test firings can lead to very high surface temperatures on the flame deflector. Surface temperatures on the flame deflector are usually controlled through the use of carefully designed cooling hole patterns which are fed by an industrial water supply. The interaction of the impinging plume with water from the cooling nozzles determines the optimal spacing and size of the cooling nozzles as well as the adequacy of cooling water mass flow rates. In the past, un-cooled rocket plume analysis were overlaid with "experience" curves to 
determine cooling hole patterns. In this paper, a unified tightly coupled multi-phase computational framework has been described that will be used to analyze and design various spray nozzle configurations for effective cooling of the flame deflector in rocket engine test stands. This methodology will also enable detailed study of the interaction between the rocket and droplet spray plumes as well as the entrainment of cold air into the test facility. Verification and bench-marking of the scheme was shown through test cases with incresing levels of complexity, comprising of phase change studies of simple flowfields with droplets, and cooling of a heated plate/chamber with spray nozzle. The validity of the method was demonstrated for a sub-scale test facility for which the computational framework was accurately able to replicate the experimental observations of hot spots on the flame deflector. Future work will include consideration of other spray hole configurations on the sub-scale facility as well as application to a full-scale test facility.

\section{Acknowledgments}

The CRAFT Tech authors acknowledge funding for this work through a NASA SBIR, Contract Number NNX08CA36C funded by NASA Stennis Space Center. Dr Harry Ryan was the project monitor and has provided important guidance and helpful suggestions during the course of this project. We would also like to thank Mr. David Coote of NASA Stennis Space Center for his recommendations and suggestions.

\section{References}

1. Herring, Mack R. Way Station to Space: A History of the John C. Stennis Space Center. NASA SP-4310, 1997.

2. Allgood, D. C. and Ahuja, V., "Computational Plume Modeling of Conceptual ARES Vehicle Stage Tests," AIAA-20075751, 43 ${ }^{\text {rd }}$ AIAA/ASME/SAE/ASEE Joint Propulsion Conference and Exhibit, Cincinnati, OH, July 8-11, 2007.

3. Kenzakowski, D. C., and Brinckman, K.W., "CFD Simulations of NASA B-2 Spray Chamber During Rocket Fire," 46 ${ }^{\text {th }}$ Aerospace Sciences Meeting and Exhibit, AIAA Paper No. AIAA-2008-0790, Reno, NV, 7-10 Jan 2008.

4. Henderson, C.B., "Drag Coefficients of Spheres in Continuum and Rarefied Flows," AIAA Journal, Vol. 14, No. 6, page 707, 1979.

5. Hermsen, R.W., "Review of Particle Drag Models," JANNAF Performance Standardization Subcommittee 12th Meeting Minutes, CPIA Publication, January 1979.

6. Ahuja, V., Hosangadi, A., Mattick, S., Lee, C.P., Field, R.E. and Ryan, H., "Computational Analyses of Pressurization in Cryogenic Tanks", AIAA Paper No. AIAA-2008-4752, 44th AIAA/ASME/SAE/ASEE Joint Propulsion Conference\& Exhibit, Hartford, CT, July 2008.

7. Hosangad, A., Lee, R. A., York, B. J., Sinha, N., and Dash, S. M., "Upwind Unstructured Scheme for Three-Dimensional Combusting Flows," Journal of Propulsion and Power, Vol. 12, No. 3, May-June, 1996, pp. 494-503.

8. Hosangad, A., Lee, R. A., Cavallo, P. A., Sinha, N., and York, B. J., "Hybrid, Viscous, Unstructured Mesh Solver for Propulsive Applications," AIAA-98-3153, 34 ${ }^{\text {th }}$ AIAA/ASME/SAE/ASEE Joint Propulsion Conference and Exhibit, Cleveland, OH, July 13-15, 1998.

9. Hosangad, A., Cavallo, P. A., Arunajatesan, S., Ungewitter, R., and Lee, R. A., "Aero-Propulsive Jet Interaction Simulations Using Hybrid Unstructured Meshes," AIAA-99-2219, 35 th AIAA/ASME/SAE/ASEE Joint Propulsion Conference and Exhibit, Los Angeles, CA, June 20-24, 1999.

10. Barth, T. J., "Numerical Aspects of Computing Viscous High Reynolds Number Flows on Unstructured Meshes," AIAA Paper 91-0721, Jan. 1991.

11. Barth, T. J., and Linton, S. W., "An Unstructured Mesh Newton Solution for Compressible Fluid Flow and Its Parallel Implementation," AIAA Paper 95-0221, 1995.

12. Saurel, R., Daniel, E., Loraud, J. C., "Two-phase flows: second-order schemes and boundary conditions," AIAA Journal, Vol 32, pp 1214-1221, 1997.

13. Slater, S. A., and Young, J. B., "The calculation of inertial particle transport in dilute gas-particle flows, International Journal of Multiphase Flows, Vol. 27, pp61-87, 2001.

14. Saito, T, Marumoto, M, Takayama, K., "Numerical investigations of shock waves in gas-particle mixtures," Shock Waves, Vol. 13, pp 299-322, 2003.

15. Igra, O, Hu, G, Falcovitz, J., and Wang, B. Y., "Shock wave reflection from a wedge in a dusty gas," International Journal of Multiphase Flow, Vol. 30, pp 1139-1169, 2004.

16. Sachdev, J. S., Groth C. P. T, and Gottlieb, J. J., "Numerical solution scheme for inert, disperse, and dilute gas-particle flows,"International Journal of Multiphase Flow, Vol. 33, pp 282-299, 2007.

17. Ciofala, M., Di Piazza, I., and Brucato, V., "Investigation of the cooling of hot walls by liquid water sprays," International Journal of Heat and Mass Transfer, Vol 42, pp 1157-1175, 1999. 